\title{
Electromagnetic Interference of Switching Power Supplies and Corresponding Restraining Measures
}

\author{
Shusen Meng \\ Jiangsu Vocational Institute of Commerce, Nanjing, Jiangsu Province, China \\ mengshusen@126.com
}

Keywords: switching power supply; electromagnetic interference; restrain; filtering; shielding; ground connection.

\begin{abstract}
Starting from the mechanism of electromagnetic interference, this paper analyzes the causes of electromagnetic interference in switching power supplies, and summarizes measures which can restrain the electromagnetic interference. Effective restraining measures include absorption circuit, soft-switching technology, filter technique, as well as shielding and grounding technology.
\end{abstract}

\section{Introduction}

The power supply is essential for all kinds of electronic equipment. The performances of power supplies directly relate to the technical indexes, as well as the safely and reliably of equipment. At present, commonly used DC regulated power supplies can be divided into two types: linear power supplies and switching power supplies. The switching power supply is known for its energy saving and high efficiency; it represents the development direction of regulated power supplies. Now it has become the mainstream product in the regulated power supply market. Switching power supplies are widely used in communication, control, computer and other fields on account of their advantages like small size and high power factors. Most switching power supplies adopt the technology of pulse width modulation, which is featured by high frequency, high efficiency and high power density. However, because of its high frequency on-off mode, the process of fast transient generates electromagnetic interference itself; the electromagnetic interference directly affects the normal operation of the electronic equipment. Therefore, in the process of developing and designing switching power supplies, it is an important topic to suppress the electromagnetic interference of switching power supplies and improve its anti-interference ability, in order to ensure the long-term safety and reliable operation of equipment.

\section{Mechanism of Electromagnetic Interference in Switching Power Supplies}

\subsection{Basic rectifying process of rectifiers}

The basic rectification process is the most common cause of electromagnetic interference. This is because, after passing the rectifier, sinusoidal currents become one-way pulse currents; they are not single frequency currents any longer. According to Fourier series, the current wave can be divided into DC component and the sum of a series frequency of fundamental components and harmonic components with different times. The high order harmonics can produce conducted interference and radiation interference along the transmission line. On one hand, the current waveform which is connected to the front of power line is distorted; on the other hand, it generates radio frequency interference through the power line.

\subsection{Interference caused by the reverse recovery time of diode}

In high frequency rectifying circuit, large positive currents flow through the rectifier diode during positive onset. Under the influence of reverse voltage, a lot of carriers stop and accumulate in PN junction. Before these carriers disappear, the currents flow reversely. Large current changes occur 
due to the dramatically decrease of reverse recovery current.

\subsection{Harmonic interference produced by working state switching tubes}

Large pulse currents flow through the power switch tube in connectivity. For example, the input forward, push-pull and bridge converter currents are approximately rectangular waves in resistive loads, and are rich in high-order harmonic components. In addition, abrupt changes caused by the leakage inductance of high frequency transformer can also lead to peak interference when the power switch tube is switching off.

\subsection{High frequency transformer}

High frequency transformer is used as the isolation and voltage variation in switching power supplies. But in cases of high frequency, the isolation is incomplete. The distributed capacitance between transformer layers makes the high-frequency noises of switching power easily transfer between primary and secondary stages. If the capacitance filter capacity is insufficient, or the high frequency characteristic is not good, the high frequency impedance on capacitor will make high-frequency current transmit to the alternating current power supply through differential mode and generate conducted interference. Besides, the distribution capacitance of transformer to the outer shell can form another high frequency path; thus, the electromagnetic field around the transformer can couple with other lead wires easily, and lead to interference.

\subsection{Other reasons}

The printed circuit board (PCB) line usually adopts manual layout with great randomness. The high near field interference of PCB, and the improper location of printing plate device can also cause electromagnetic interference. Other reasons include parasitic components and imperfect principle diagram design of switching power supplies.

\section{Restraining Measures of Electromagnetic Interference in Switching Power Supplies}

Electromagnetic compatibility has three elements. We can start from three aspects to solve the problem of electromagnetic interference of switching power supplies. Basic measures include reducing interference signals generated by the interference source; cutting off the transmission way of interference signals; enhancing the anti-interference ability of the body. Therefore, main methods to suppress the electromagnetic interference of switching power supplies include circuit structure, soft switching technology, filtering technique, shielding technology and reasonable anti-interference PCB design.

\subsection{Circuit structure}

Absorption circuit. The main reason of EMI generation in switching power supplies is the rapid change of voltage and current. So it is necessary to reduce the change rates of voltage and current in the circuit. The absorption circuit can inhibit EMI. Through providing bypass when switching off, it absorbs energy stored in parasitic distributed parameters, so as to suppress interference. Specifically, the RC absorption circuit or the D-RC absorption loop can be parallel connected to both ends of the switch tube. Thus, the circuit can absorb high surging peak voltage in the switch tube when switching on and off, and reduce the interference of the switch circuit.

Soft-switching technology. Traditional AC/DC full-wave rectifier circuits use rectification and capacitor filtering. A circuit is the combination of nonlinear devices and energy storage elements. The waveform of input voltage is sinusoidal, but it is distorted and become pulse wave. The resulting harmonic current has harmful effects on the power grid, and brings down the input power factor of the current. The use of active power factor correction (APFC) circuit in rectifying circuit can avoid these shortcomings as in Figure 1. Unlike typical PFC main circuits, this circuit selects the lossless absorption buffer network. The network reduces the switching loss of the switch tube, improves its stability and prolongs its service life. It uses a set of passive components to achieve zero current turning on and zero voltage turning off, which improves the working efficiency of power supplies, 
and reduces the production cost compared with other resonant soft switching circuits.

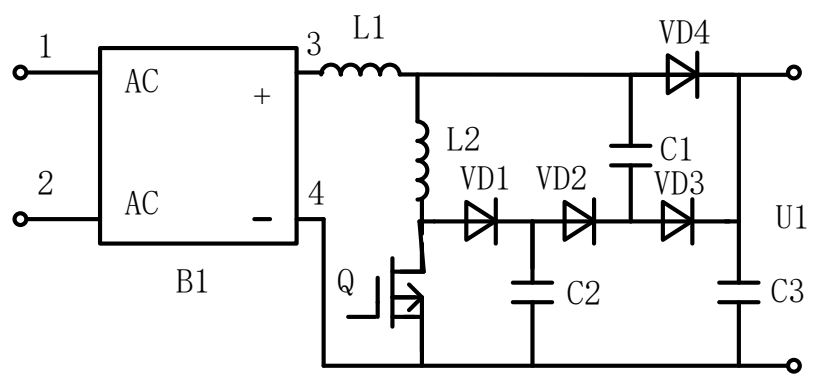

Figure 1. PFC lossless absorption circuit

\subsection{Filtering technique}

The filtering technique is an effective way to restrain the electromagnetic interference of switching power supplies, especially the conduction interference and a part of radiation interference. The interference signal is injected into the public grid from the input of power supplies and generates conductive interference. There are two forms of conducted interference: common mode interference and differential mode interference. Generally speaking, differential mode interference has small amplitude and low frequency; it produces little interference. Common mode interference has large amplitude and frequency, and may cause radiation interference through the power cord. Therefore, an anti- electromagnetic interference (EMI) filter should be installed at the power input terminal. The EMI filter should be installed between the power supply line and the switching power supply. It only permits the passing of power current, and plays an important role in improving the reliability of switching power supplies. A commonly used power filter is shown in Figure 2. In this picture, filter capacitors C1, C2, C3, C4 and inductor L1, L2 constitute a common modefilter, which is used to filter common mode interference. C0 and C5 are high frequency bypass capacitors; they form a differential modefilter with inductor L1 and L2. This mode can be used to filter differential mode interference.



Figure 2. EMI filter

Shielding and grounding technology. Shielding is an effective way to suppress the radiation interference of switching power supplies. Two general categories of shielding are electrostatic shielding and electromagnetic shielding. Electrostatic shielding is mainly used to prevent the influence of static electric field and constant magnetic field. Electromagnetic shielding is mainly used to prevent the influence of alternating electric field, magnetic field and alternating electromagnetic field. Large coupling capacitance exists between the high frequency transformer coils in the switching power supplies. The capacitance can be reduced by the Faraday shield in the transformer structure when the shielding layer is grounded. In addition, photoelectricity transformer can also be used to cut off noise coupling and resist interference.

Grounding means designing a conductive path between a selected point of the system and a certain ground plane. Good grounding can not only guarantee the safety of the people and equipment, but also resist electromagnetic interference. The correct combination of grounding and shielding can solve most of the EMI problems.

PCB layout. Improper PCB board layout is an important reason of interference. The components layout should satisfy the requirements of mechanical structure of the system firstly, and locate all devices which should be strictly positioned (such as transformers, sensors, radiators and monitors) properly. Some larger devices cannot be directly installed on PCB; they should be supported and 
installed on the shell. However, from the consideration of electromagnetic compatibility, the layout of components should follow several common principles. Some sensitive devices, such as phase locked loops, are particularly sensitive to noise interference; horseshoe shaped insulating grooves should be etched in the copper foil of surrounding power supplies. Connectors and pins should be positioned according to the location of components. It is the best to put all connectors on one side of the printed circuit board, so as to avoid common mode current radiation. I/O driver should be close to the connector, in order to avoid long lines of I/O signal on board and unnecessary interference signal coupling.

\section{Conclusion}

Switching power supply technology is a kind of comprehensive technology. It attracts increasingly attentions in the aspects of electromagnetic interference and electromagnetic compatibility with other electronic devices. A variety of means must be adopted to effectively restrain the electromagnetic interference produced by switching power supplies, as well as enhance the electromagnetic compatibility and extend the potential usage of switching power supplies.

\section{References}

[1] L.F. Zhou, et al., EMI power filter design and simulated analysis, J, Low Voltage Apparatus. 2004.

[2] T. Xu, Analysis of electromagnetic interference in switching power supply and restrain, J. Journal of Hehai University Changzhou. 6 (2006).

[3] Y.M. Li, et al., Simulation study of electromagnetic interference in switching power supply, J. Journal of Hunan Institute of Science and Technology (Natural Sciences). 3 (2012).

[4] A.J. Jin, et al., Analysis and design application of switching power supply EMC, J. Information Technology. 9 (2014)

[5] Z.J. Bai, Electromagnetic interference of switching power supply and restrain technology, J. Science and Technology \& Innovation. 1 (2016). 